\title{
Cerebral vasoconstriction reactions and plasma levels of ETBR, ET-1, and eNOS in patients with chronic high altitude disease
}

\author{
SHIZHENG WU $^{1}$, GUISHENG HAO ${ }^{1}$, SHUKUN ZHANG $^{2}$, \\ DONGMEI JIANG ${ }^{3}$, TANA WUREN ${ }^{3}$ and JUNMING LUO ${ }^{2}$ \\ Departments of ${ }^{1}$ Neurology and ${ }^{2}$ Pathology, the Qinghai Provincial People's Hospital, Xining, Qinghai 810007; \\ ${ }^{3}$ Research Center for High Altitude Medicine, Qinghai University, Xining, Qinghai 810001, P.R. China
}

Received January 7, 2016; Accepted July 27, 2016

DOI: $10.3892 / \mathrm{mmr} .2016 .5555$

\begin{abstract}
The aim of the present study was to examine cerebral vasoconstriction in patients with chronic high altitude disease [cerebrovascular reactivity (CVR)], and to evaluate differences in alterations of brain vascular contractile reactivity of chronic mountain sickness (CMS) patients and healthy controls. Alterations of endothelin (ET) and its receptor, as well as endothelial nitric oxide synthase (eNOS) levels in the plasma were examined to determine the cerebral reservation capacities in CMS patients. Transcranial Doppler ultrasound and carbon dioxide analysis methods were used to detect the CVR variances. At the same time, enzyme-linked immunosorbent assay approaches were utilized to detect the ET and ET B receptor and the eNOS levels in serum of the CMS patients and healthy controls. CVR and CVRI levels in CMS patients were lower than those of the healthy control subjects and the difference was statistically significant $(\mathrm{P}<0.05)$. By contrast, eNOS and ET-1 levels were not statistically significant for CMS and healthy controls $(\mathrm{P}>0.05)$. However, the ET receptor concentration level was higher in CMS than the healthy controls. Thus, ET-1 may not be a direct etiological variation but may play compensatory roles in CMS patients. The results of the study may provide scientific clues for the prevention and treatment of CMS with higher blood coagulation states of cerebral infarction in patients with chronic high altitude disease.
\end{abstract}

\section{Introduction}

Red blood cell (RBC) excessive hyperplasia and significant pulmonary hypertension are the main pathophysiological manifestations of chronic mountain sickness (CMS) in plateau

Correspondence to: Dr Shizheng Wu, Department of Neurology, Qinghai Provincial People's Hospital, 2 Gonghe Road, Xining, Qinghai 810007, P.R. China

E-mail: shizheng_wu1@163.com

Key words: chronic mountain sickness, cerebrovascular reactivity, endothelin-1, endothelin B receptor, endothelial nitric oxide synthase areas (1). Hypoxemia is a significant clinical characteristic thereof (2). The neuron is the most oxygen reaction-sensitive cell. Long-term chronic hypoxemia seriously affects normal physiological functions, thus cerebrovascular produces reflective factors to improve the blood supply of neurons under chronic hypoxia (3). Cerebrovascular reactivity (CVR) refers to cerebrovascular pathological factor stimulation by contracting or diastolic change to achieve stable regional cerebral blood flow (CBF) and cerebral perfusion (4), and the ability of reaction is an important index of cerebral reserve capacity. Thus, CVR is used as an evaluation standard of testing real-time brain blood supply.

The Transcranial Doppler (TCD) test is becoming a CVR method and has been extensively applied in clinical research during recent years (5). In previous studies, alteration of arterial blood pressure of carbon dioxide $\left(\mathrm{CO}_{2}\right)$ from subjects having to hold their breath were used to detect cerebral arterioles vasomotor responses (6). As this method is unable to accurately record the change of the duration of holding breath and $\mathrm{pCO}_{2}$, the use has been gradually reduced in the clinic. At present, most scientists use synchronous methods to detect the $\mathrm{CBF}$ velocity and $\mathrm{pCO}_{2}$, including quiet breathing, low carbonate platform stage and/or hypercapnia platform stage data, respectively (7). The collected data are more accurate and straightforward with fewer interference factors. In the present study, a DWL company Multi-Dop X TCD ultrasound monitor together with a $\mathrm{CO}_{2}$ monitoring module were used to accurately determine long-range synchronization acquisition $\mathrm{CO}_{2}$ partial pressure, breathing graph and detect changes in middle cerebral artery blood flow and spectrum.

Endothelin (ET) is a 21 amino acid bioactive peptide that was separated and purified from pig aortic endothelial cells by Yanagisawa et al (8). The peptide is divided subtypes according to the molecular structure: ET-1, ET-2 and ET-3, respectively. ET-1 is the only one existing vascular endothelial cell with strong biological effects, and the most production, while ET-2 and ET-3 are mainly expressed in the brain, kidney, adrenal gland, and small intestine (9). ET and its receptor have a strong and lasting vascular contraction effect.

ET receptor (ETR) can be divided into ET B receptor (ETBR) and ETAR, which are mainly distributed in vascular smooth muscle, cardiac muscle cells and fibroblasts (9). Strong vasoconstrictor and cell proliferation occur when it binds to 
ET. ETBR is subdivided into ETBR1 and ETBR2 subtypes, which are mainly distributed in vascular endothelium, with a small amount of distribution in vascular smooth muscle. ET binds ETBR and increases nitric oxide (NO) secretion by endothelial cells, while prostaglandins play a role in diastolic blood vessels (10).

NO synthase (NOS) is divided into neuronal NOS (nNOS), inducable NOS and endothelial NOS (eNOS). The nNOS and eNOS are mainly expressed in cerebrovascular endothelial cells (11). eNOS can produce NO gas in the angiogenesis effects of diastolic heart failure (12). eNOS activates hypoxia-induced factor-1 (HIF-1) and HIF-2, and promotes endothelial cells to produce more NO gas during hypoxia, which can stimulate the synthesis of soluble guanylate cyclase acid [cyclic guanosine monophosphate (cGMP)] in cytoplasm, leading to vasodilation by way of free diffusion into vascular smooth muscle cells (VSMCs) (13).

Chronic cerebrovascular diastolic and condensation reaction under hypoxia are considered controversial issues. Patients with chronic plateau CVR studies have yet to be reported. The aim of the present study was to use TCD accompanied with $\mathrm{CO}_{2}$ (14) to evaluate CVR and to determine the change in plasma content of ET, ETBR and eNOS in order to examine the differences in cerebral circulation reserve capacity of individuals residing at an altitude of 3,600 $\mathrm{m}$ and the healthy controls residing at low attitude. In addition, scientific data for the prevention and treatment of cerebral infarction in patients with chronic plateau condition with high blood clot were provided.

\section{Materials and methods}

Healthy controls. A total of 23 male healthy volunteers, with an average age of $34.4 \pm 15.6$ years, residing at an altitude of 2,200 $\mathrm{m}$, were selected from the Qinghai Xining region between September and November, 2014, at the Qinghai Provincial People's Hospital Medical Center. Inclusion criteria for the study were: i) Patients were 18-60 years of age; ii) volunteers did not have any chronic medical history including hypertension, diabetes, hyperlipidemia and heart, or cerebrovascular disease; iii) lack of lifestyle habits such as drinking or smoking; iv) the blood flow spectrum was clearly evident in the temporal area during the process of TCD examination; and v) evidence of TCD blood flow turbulence, eddy current signal and rough vascular murmur.

The study was approved by the Qinghai Provincial People's Hospital Ethics Committee. Investigators were informed of the related purpose and method of tests. Written informed consent was provided by the participants.

CMS patients. A total of 26 male patients with chronic altitude disease, with an average age of $32.8 \pm 16.2$ years, were selected at the Physical Examination Center of Yushu People's Hospital (Qinghai, China). CMS diagnosis was made according to the 6th International Plateau Hypoxia Physiological Academic conference using the chronic plateau disease international diagnostic criteria, i.e., 'Qinghai Standard' (2004). The main criterion included erythrocytosis (female hemoglobin $190 \mathrm{~g} / \mathrm{l}$, or male hemoglobin or $210 \mathrm{~g} / \mathrm{l})$ (1). The chronic plateau disease clinical symptoms were alleviated gradually, when patients descended from a high altitude hypoxic environment, and while the clinical symptoms appeared again when the patients became exposed to the plateau environment anew. The inclusion criteria were: i) The 'standard' in Qinghai score $>6$; ii) 18-60 years of age; iii) exclusion of patients with hypertension, diabetes, hyperlipidemia, cerebrovascular disease, acute and chronic disease and heart disease; iv) no long-term drinking, smoking or other unhealthy lifestyle habit; and v) presenting with TCD blood flow turbulence, vortex signal and rough vascular murmur. Exclusion criteria were: i) TCD examination did not clearly show the blood flow spectrum; and ii) the patients were reluctant to cooperate.

The scoring method of the chronic altitude sickness 'Qinghai Standard' was used to score symptoms as follows: Asthma and palpitations (0, without asthma/palpitations; 1, mild asthma/palpitations; 2, moderate asthma/palpitations; and 3, severe asthma/palpitations); insomnia (0, normal; 1 , cannot normally sleep; 2 , lack of sleep; and 3, could not follow sleep); cyanosis ( 0 , normal; 1 , light; 2 , moderate; and 3, severe); vasodilation ( 0 , normal; 1 , mild; 2 , moderate; and 3 , severe); paraesthesia $(0$, normal; 1 , mild; 2 , moderate; and 3 , severe) headache $(0$, no; 1 , mild; 2 , mild; and 3 , severe) tinnitus $(0$, without; 1 , mild; 2 , mild; and 3 , severe) hemoglobin concentration (men: $180 \mathrm{~g} / \mathrm{l}<\mathrm{Hb}<210 \mathrm{~g} / 1 \mathrm{0}, \mathrm{Hb} 210 \mathrm{~g} / 1$ or $>3$; women: $160 \mathrm{~g} / \mathrm{l}<\mathrm{Hb}<190 \mathrm{~g} / 1 \mathrm{O}, \mathrm{Hb} 210 \mathrm{~g} / 1$ or $>3$ ). According to the above symptoms and the level of hemoglobin concentration, chronic disease of plateau was divided into no chronic plateau sickness, 0-5; mild chronic plateau sickness, 6-10; moderate chronic disease of plateau, 11-14; and severe chronic plateau sickness, $>15$.

Blood serum analysis and enzyme-linked immunosorbent assay (ELISA). Peripheral blood (4 ml) was obtained for detection (HF-3800 automatic blood cell analyzer) of the RBC count, hemoglobin concentration, $\mathrm{RBC}$ deposit, and to determine blood oxygen saturation using a portable oxygen saturation device. The remaining supernatant was collected after centrifugation at $2,800 \mathrm{x} \mathrm{g}$ for $10 \mathrm{~min}$, and was preserved at $-80^{\circ} \mathrm{C}$ freezer prior to subsequent analysis. ELISA was performed according to kit instructions (ETBR: Anti-EDNRB ELISA kit; OriGene Technologies, Inc., Rockville, MD, USA. eNOS: eNOS ELISA kit; Cell Signaling Technology, Boston, MA, USA. ET: ET-1 ELISA kit; R\&D Systems, Inc., Minneapolis, MN, USA).

TCD detection. TCD monitor (Multi-DopX DWL Co.) was used to study CVR (15). The patients were asked to sit in calm state (breathing rate of 16-20 times/min) in supine position. Two ultrasonic probes with a head frame (frequency $2 \mathrm{MHz}$ ) were used to make contact with the bilateral temporal of subjects, with 55-65 $\mathrm{mm}$ probing depth, and the speed and direction of bilateral middle cerebral artery and state of spectrum were detected. The patients were asked to wear a mask, and were recorded while breathing quietly for $2 \mathrm{~min}$, followed by hyperventilation for $2 \mathrm{~min}$, calm state breathing for $2 \mathrm{~min}$, autologous $\mathrm{CO}_{2}$ inhalation for $2 \mathrm{~min}$ (after $2 \mathrm{~m}$ long extension tube) followed by calm state breathing for $2 \mathrm{~min}$, and holding breath (patients instructed to inhale and hold breath for as long as possible after a deep breath) for 2 min to quiet breathing parameters. Each baseline was selected and its largest middle cerebral artery (MCA), mean CBF velocity and average $\mathrm{ETCO}_{2}$ were 
Table I. General characteristics of the research subjects (mean \pm standard deviation).

\begin{tabular}{|c|c|c|c|c|c|c|}
\hline Groups & No. of cases & $\mathrm{Hb}^{\mathrm{a}}(\mathrm{g} / \mathrm{l})$ & WBC (x109/1) & $\operatorname{PLT}\left(\times 10^{9} / 1\right)$ & $\mathrm{HCV}^{\mathrm{b}}(\%)$ & $\mathrm{SaO}_{2}^{\mathrm{c}}(\%)$ \\
\hline Control & 23 & $165.7 \pm 8.9$ & $5.9 \pm 1.6$ & $189.6 \pm 35.0$ & $55.7 \pm 6.9$ & $87.4 \pm 3.5$ \\
\hline CMS & 26 & $220.6 \pm 15.3$ & $6.8 \pm 1.5$ & $179.1 \pm 46.0$ & $71.9 \pm 5.7$ & $77.9 \pm 6.9$ \\
\hline t-value & & 26.464 & 2.896 & 1.302 & 12.703 & 8.934 \\
\hline P-value & & 0.000 & 0.002 & 0.098 & 0.000 & 0.000 \\
\hline
\end{tabular}

${ }^{\mathrm{a}} \mathrm{Hb}$, hemoglobin; ${ }^{\mathrm{HCV}}$, hematocrit; ${ }^{\mathrm{S}} \mathrm{SaO}_{2}$, oxygen saturation; two groups: $\mathrm{P}<0.01$. WBC, white blood cell; CMS, chronic mountain sickness; PLT, platelet cell count.

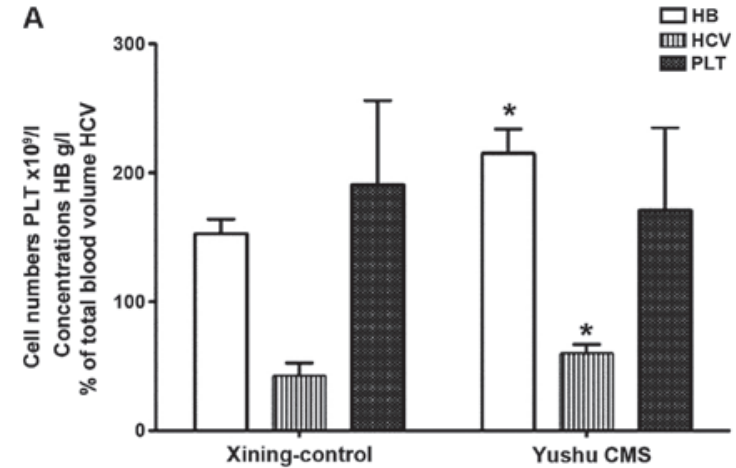

B

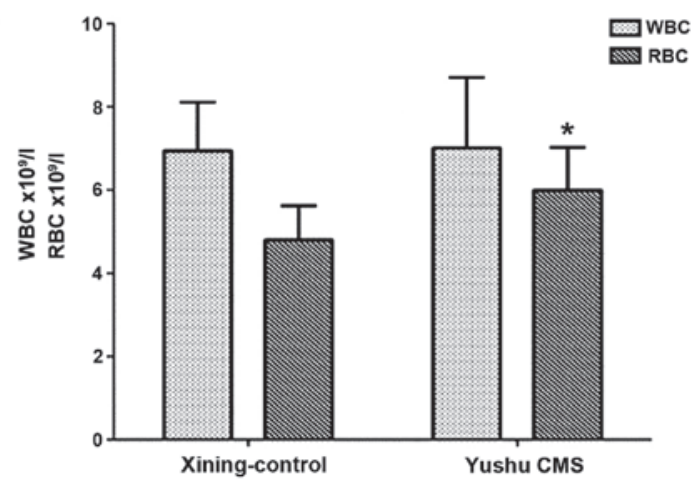

Figure 1. Platelet cell count (PLT), Hepatitis B (HB), blood volume (HCV), white blood cell (WBC) and red blood cell (RBC) count for healthy controls and chronic mountain sickness (CMS) patients. (A) HB, HCV and PLT; (B) WBC and RBC. "P $<0.05$, compared with Xining control.

recorded. The formulae used for the various parameters were: $\mathrm{CVR}=(\mathrm{V}$ changed-V baseline $) /\left(\mathrm{ETCO}_{2}\right.$ change $-\mathrm{ETCO}_{2}$ baseline $)$, and CVRI = (V change-V baseline $) / \mathrm{V}$ baseline $\times 100 \% /$ $\mathrm{ETCO}_{2}$ change-ETCO${ }_{2}$ baseline), where $\mathrm{V}$ was the baseline for breathing in a calm state, MCA average, $\mathrm{CBF}$ velocity and the average of $\mathrm{ETCO}_{2}$. V changed after 2 min for autologous $\mathrm{CO}_{2}$ inhalation, MCA mean, $\mathrm{CBF}$ velocity and the average $\mathrm{ETCO}_{2}$. According to the exhaled gas $\mathrm{CO}_{2}$ partial pressure and blood flow parameters of MCA, CVR and CVRI were calculated. In the abovementioned formulae, the CVR calculation constituted the CVR absolute slope (absolute slope), indicating that alterations in the $\mathrm{mm} \mathrm{hg} \mathrm{CO}_{2}$ partial pressure corresponded to the absolute value of blood flow velocity, whereas under the influence of the baseline flow velocity, the difference between the data were more obvious. CVRI referred to the result of the relative slope (relative slope), indicating changes in $\mathrm{mm} \mathrm{hg} \mathrm{CO}_{2}$

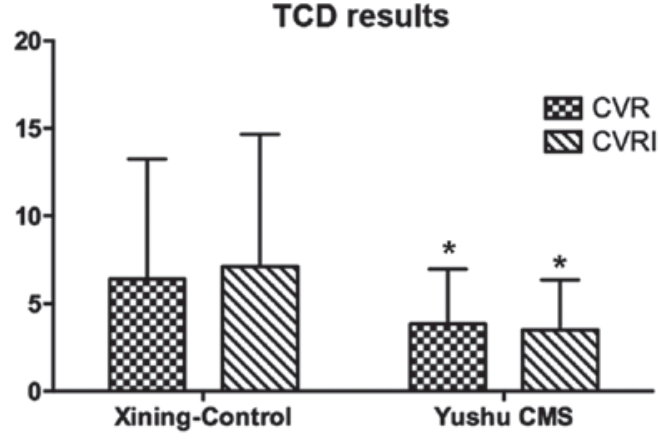

Figure 2. Levels of cerebrovascular reactivity (CVR) and CVRI between controls and chronic mountain sickness (CMS) patients. TCD, transcranial Doppler. ${ }^{*} \mathrm{P}<0.05$, compared with Xining control.

partial pressure corresponded to changes in the percentage of blood flow velocity, providing an objective description of the degree of change in $\mathrm{CBF}$ velocity with $\mathrm{CO}_{2}$ partial pressure.

Statistical analysis. Data were analyzed using SPSS 17.0 statistical software (SPSS, Inc., Chicago, IL, USA). Measurement data were presented as mean \pm standard deviation (using two independent samples t-test, inspection level $\alpha=0.05$. $\mathrm{P}<0.05$ was considered to indicate a statistically significant difference.

\section{Results}

Blood and $\mathrm{SO}_{2}$. The CMS group comprised 26 males, with an average age of $34.4 \pm 15.6$ years while the healthy controls comprised 23 males, with an average age of $32.8 \pm 16.2$ years. There was no statistically difference in average age composition and comparable $(\mathrm{P}>0.05)$. Patient characteristics are shown in Table I and various cell counts are shown in Fig. 1A and B.

Comparison of CVR, CVRI and eNOS, ET-1, and ETBR. The cerebrovascular response values of CMS patients were examined, and it was found that CVR and CVRI were significantly lower compared to the healthy controls (Fig. 2). The serum levels of eNOS and ET-1 in the CMS group increased slightly, albeit there was no statistical difference compared to the healthy control group ( $\mathrm{P}>0.05$ ) (Fig. 3A). The quantity of ETBR expression in the CMS group was significantly higher than the control group. The difference was statistically significant $(\mathrm{P}<0.05)$ (Fig. 3B and Table II). 
Table II. Comparison of levels of CVR, CVRI, eNOS, ET-1 and ETBR values in CMS and healthy controls.

\begin{tabular}{lcccccc}
\hline Group & No. of cases & CVR & CVRI & eNOS $(\mu \mathrm{g} / \mu \mathrm{l})$ & ET $(\mu \mathrm{mol} / \mathrm{l})$ & ET-RA/R $(\mathrm{pg} / \mathrm{ml})$ \\
\hline CMS & 26 & $3.8 \pm 3.0$ & $3.5 \pm 2.9$ & $39.5 \pm 10.8$ & $42.0 \pm 20.3$ & $386.1 \pm 181.6$ \\
Control & 23 & $6.4 \pm 6.9$ & $7.1 \pm 7.5$ & $38.3 \pm 13.9$ & $41.9 \pm 17.9$ & $312.3 \pm 138.1$ \\
t-value & & $2.336^{\mathrm{a}}$ & $3.084^{\mathrm{a}}$ & 0.474 & 0.039 & $2.317^{\mathrm{a}}$ \\
P-value & & 0.011 & 0.001 & 0.325 & 0.484 & 0.011 \\
\hline
\end{tabular}

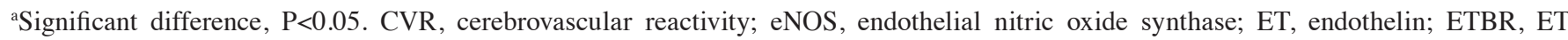
receptor B; CMS, chronic mountain sickness.
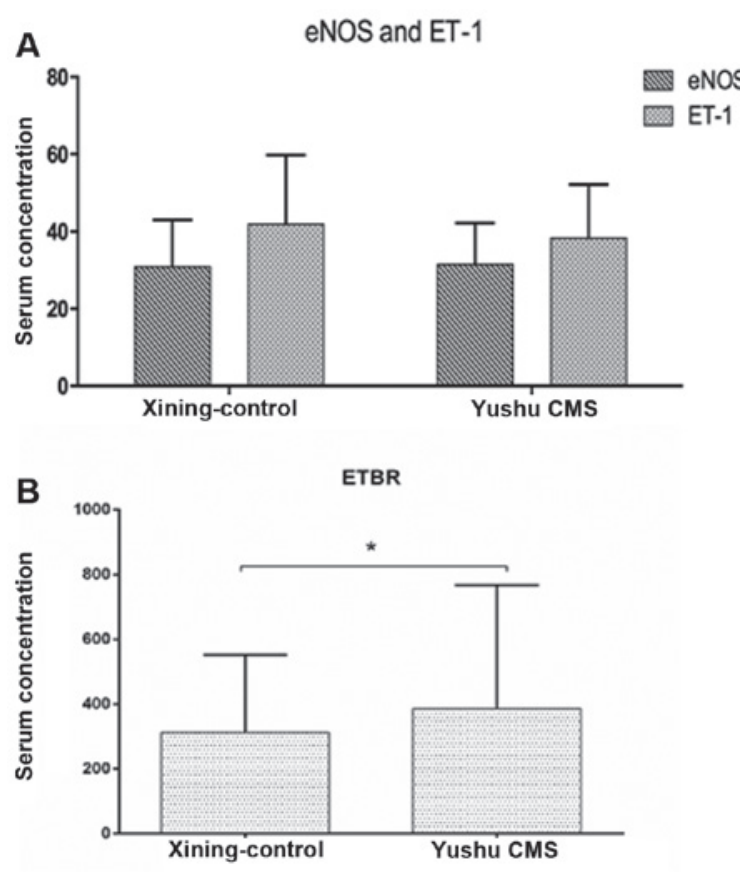

Figure 3. Levels of endothelial nitric oxide synthase (eNOS), endothelin (ET)-1 between controls and chronic mountain sickness (CMS) patients, were found to be non-significant $(\mathrm{P}>0.05)$ (panel A). The level of ET receptor $\mathrm{B}$ ETBR show a significant difference in CMS patients in Yushu compared to the healthy controls in Xining $(\mathrm{P}<0.05)$ (panel B).

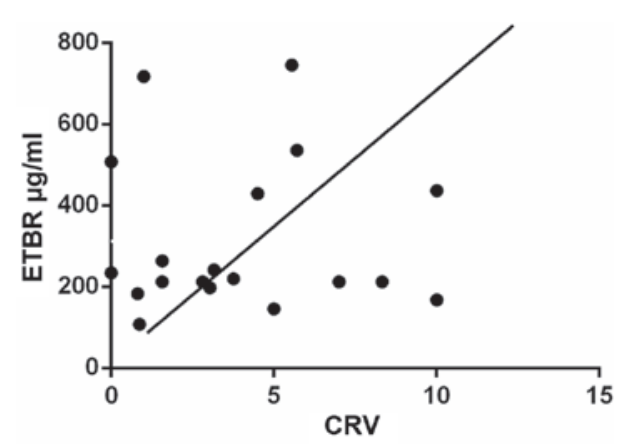

Figure 4. Correlation between the concentration of endothelin receptor $\mathrm{B}$ (ETBR). Cerebrovascular reactivity (CVR) level for chronic mountain sickness group was 0.378 .

Correlation analysis results. No correlation of the content of serum levels of ET-1 and ETBR and CVR $(\mathrm{P}>0.05)$ were observed in the healthy control group. No correlation was observed between the content of ET-1 and CVR $(\mathrm{P}>0.05)$, while an obvious correlation was evident between the ETBR and CVR content. The r-value was 0.378 for the CMS group (Fig. 4).

\section{Discussion}

The molecular mechanisms of chronic plateau sickness are not clear at present. Previous findings have shown that in a high-altitude environment, the cold, low pressure, and low oxygen under intense stimulation experienced can cause pituitary-adrenal medulla hyperfunction and increases of aldosterone and $\mathrm{ADH}$, which can cause increasing peripheral resistance, sodium and water retention, and at the same time cause polycythemia (16). Excessive RBC can cause significantly increased hematocrit, blood viscosity and blood flow resistance, slowing the blood flow and sedimentation, and tissue and blood are discharged (17). Thus, the patients with chronic plateau intracranial ischemia and infarction identified were more than the healthy controls. Studies on cerebrovascular hemodynamic for CMS patients are rare at present (18). The aim of the present study was to obtain cerebrovascular response data of CMS patients to examine the effect of the plateau region on these patients including stroke, and determine cardiovascular events that may be useful in investigating this issue (19). In the present study, we found that the number of RBCs, RBC deposits and hemoglobin levels were significantly higher in CMS patients than the healthy controls. At the same time, $\mathrm{SaO}_{2}$ was lower in CMS compared to the healthy controls. There was obvious hypoxemia in CMS patients, indicating excessive proliferation of RBCs and obvious hypoxemic clinical manifestations in CMS patients (20). However, the mechanism of mutual influence and RBCs of the CBF remains to be clarified, although the existing literature offers the following as possible reasons: i) The blood plasma viscosity altered the viscous friction force causing an increase in cerebral vascular resistance; and ii) arterial blood oxygen content was reduced in CMS patients, leading to vascular relaxation response in order to maintain adequate oxygen in the brain tissue (21).

Brain tissue is an important component of the central nervous system and an organ relying primarily on oxygen to function. (4) Additionally, it is extremely sensitive to the change of blood oxygen partial pressure $\left(\mathrm{pO}_{2}\right)$. When the $\mathrm{pO}_{2}$ 
is decreased to $40-45 \mathrm{mmHg}$, it can cause cerebrovascular diastolic dysfunction (22). When the brain lacks oxygen, the feedback brain artery expands to increase the brain blood supply, but when cerebrovascular occurs at maximum expansion, its response to $\mathrm{CO}_{2}$ stimulation is obviously decreased, $\mathrm{CBF}$ velocity continues to increase and $\mathrm{pO}_{2}$ is reduced (22). Findings of previous studies have confirmed that $\mathrm{CBF}$ under the condition of response to low oxygen is a complicated pathophysiologic process that includes physical mechanisms, involved in the regulation of metabolism at biochemical and molecular levels (23). Thus, adjustment of the CBF in the plateau low-oxygen environment is complex, and cerebrovascular after a long period of low oxygen by hypoxia under chronic injury condition can lead to cerebral hemodynamic changes, thereby increasing the plateau cerebrovascular disease (13). CVR was an important indicator in the regulation of cerebrovascular diastole and contraction, which can effectively response cerebrovascular reserve force (4). The present study employed TCD, and tested the reaction of $\mathrm{CBF}$ in CMS patients and healthy controls. TCD is a non-invasive method that can effectively detect intracranial arterial blood flow dynamic change by detecting the arterial blood flow direction, blood flow velocity, frequency spectrum form, while accurately reflecting cerebral arterial stenosis, and cramps, such as ischemic pathological state (24). The present study found that CVR and CVRI were significantly reduced in CMS patients compared to the healthy controls using TCD. The results confirmed that the $\mathrm{CBF}$ reserve force was significantly decreased in CMS patients compared to the healthy controls.

Cerebral hemodynamic change may occur due to secretion vascular active factor adjustment of cerebrovascular endothelial cells (25). Previous findings suggest that, the change of CBF is controlled by vascular smooth muscle tension, which is affected by nerve, body fluid, metabolism, and various factors such as physical ones (26). Complex endothelial cells and the nerve cell network are involved in vascular contraction and relaxation factor release such as NO concentration, prostaglandins, natriuretic peptide and its receptors and ET-1, and the distribution of their receptors (27). These vessel endogenous substances can be activated under different physiological stimulations, and by changing the intracellular calcium ion concentration and regulating potassium channels cause vascular smooth muscle contraction or relaxation (28). eNOS catalytic NO synthesis and vascular factors are released. Hypoxic conditions in the HIF family induce the expression of NOS and increase downstream genes, promoting the vascular endothelial cells to produce NO. In addition, free diffusion to VSMCs stimulates the synthesis of the cytoplasm soluble guanylate cyclase acid and cGMP, resulting in vasodilatation (29). The results of the present study have shown that there was no significant difference between the concentration of ET-1 and eNOS in the CMS patients and healthy controls, indicating that the vascular regulating factor plays a role in long-term chronic hypoxia in CMS patients.

ET is coupled to phospholipase $\mathrm{C}$ with GTP-binding protein through the ETAR and ETBR receptors (23). ETAR can cause vasoconstriction mainly through VSMCs, and activated ETAR1 can promote vascular endothelial cells to release NO, which is involved in vascular diastolic and expansion (30).
However, a higher concentration of ETR, affects the expression of receptor under chronic low-oxygen stimulation, leading to a higher expression (31). The correlation analysis revealed there is no correlation between the content of ET-1 and CVR, in the CMS patients or healthy controls, while there is a significant correlation between the content of ETBR and CVR in CMS patients. However, this result shows that ETBR expression levels are associated with cerebral hemodynamics in CMS patients. A cerebral hypoxia diastolic reaction is possible due to the high expression of ETBR, rather than the result of the change of ET-1.

In conclusion, cerebral circulation reserve capacity was significantly lower in the CMS patients than the healthy controls. The level of ET-1 and eNOS plasma was not significantly different between the CMS patients and healthy controls. This finding suggests the involvement of two vascular regulating factors in patients with long-term chronic hypoxia. The high level of ETBR expression observed in CMS patients compared to healthy controls suggests that the cerebral hypoxia diastolic reaction is possible due to ETBR, rather than ET-1 itself.

\section{Acknowledgements}

This study was supported in part by Qinghai Natural Science Foundation of China (no. 2013-Z-921).

\section{References}

1. León-Velarde F, Maggiorini M, Reeves JT, Aldashev A, Asmus I, Bernardi L, Ge RL, Hackett P, Kobayashi T, Moore LG, et al: Consensus statement on chronic and subacute high altitude diseases. High Alt Med Biol 6: 147-157, 2005.

2. Javaheri S: Hypoxemia lowers cerebrovascular resistance without changing brain and blood [H+]. J Appl Physiol (1985) 60: 802-808, 1986

3. Peterson EC, Wang Z and Britz G: Regulation of cerebral blood flow. Int J Vasc Med 2011: 823525, 2011.

4. Wolff CB: Cerebral blood flow and oxygen delivery at high altitude. High Alt Med Biol 1: 33-38, 2000.

5. Vicenzini E, Ricciardi MC, Altieri M, Puccinelli F, Bonaffini N, Di Piero V and Lenzi GL: Cerebrovascular reactivity in degenerative and vascular dementia: A transcranial Doppler study. Eur Neurol 58: 84-89, 2007.

6. Harer $\mathrm{C}$ and von Kummer R: Cerebrovascular $\mathrm{CO} 2$ reactivity in migraine: Assessment by transcranial Doppler ultrasound. J Neurol 238: 23-26, 1991.

7. Markus HS and Harrison MJ: Estimation of cerebrovascular reactivity using transcranial Doppler, including the use of breathholding as the vasodilatory stimulus. Stroke 23: 668-673, 1992.

8. Yanagisawa M, Kurihara H, Kimura S, Tomobe Y, Kobayashi M, Mitsui Y, Yazaki Y, Goto K and Masaki T: A novel potent vasoconstrictor peptide produced by vascular endothelial cells. Nature 332: 411-415, 1988.

9. Simonson MS: Endothelins: Multifunctional renal peptides. Physiol Rev 73: 375-411, 1993.

10. Liu Q and Huang Z: Endothelin and cerebral vasospasm. Chongqing Med 36: 478-480, 2007.

11. Toda N and Ayajiki K: Phylogenesis of constitutively formed nitric oxide in non-mammals. Rev Physiol Biochem Pharmacol 157: 31-80, 2006.

12. Beall CM, Laskowski D and Erzurum SC: Nitric oxide in adaptation to altitude. Free Radic Biol Med 52: 1123-1134, 2012.

13. Zeng Z, Huang WD, Gao Q, Su ML, Yang YF, Liu ZC, Zhu BH: Arnebin-1 promotes angiogenesis by inducing eNOS, VEGF and HIF-1alpha expression through the PI3K-dependent pathway. Int J Mol Med 36: 685-697, 2015.

14. Kastrup A, Krüger G, Neumann-Haefelin T and Moseley ME: Assessment of cerebrovascular reactivity with functional magnetic resonance imaging: Comparison of $\mathrm{CO}(2)$ and breath holding. Magn Reson Imaging 19: 13-20, 2001. 
15. Pfefferkorn T, von Stuckrad-Barre S, Herzog J, Gasser T, Hamann GF and Dichgans M: Reduced cerebrovascular CO(2) reactivity in CADASIL: A transcranial Doppler sonography study. Stroke 32: 17-21, 2001.

16. Hackett PH and Roach RC: High-altitude illness. N Engl J Med 345: 107-114, 2001.

17. Reinhart WH, Singh A and Straub PW: Red blood cell aggregation and sedimentation: The role of the cell shape. $\mathrm{Br} J$ Haematol 73: 551-556, 1989.

18. Brugniaux JV, Hodges AN, Hanly PJ and Poulin MJ: Cerebrovascular responses to altitude. Respir Physiol Neurobiol 158: 212-223, 2007

19. Ainslie PN and Ogoh S: Regulation of cerebral blood flow in mammals during chronic hypoxia: A matter of balance. Exp Physiol 95: 251-262, 2010.

20. Qi Y, Cui S, Yang YZ, Ma Y, Li JZ and Ge R: Detection of plasma VHL, HIF-1 $\alpha$, EPO levels and polymorphism analysis of VHL 598C $>$ T in CMS. Mod Prevent Med 5: 944, 2013.

21. Lucas SJ, Burgess KR, Thomas KN, Donnelly J, Peebles KC, Lucas RA, Fan JL, Cotter JD, Basnyat R and Ainslie PN: Alterations in cerebral blood flow and cerebrovascular reactivity during 14 days at $5050 \mathrm{~m}$. J Physiol 589: 741-753, 2011.

22. Duong TQ, Iadecola $C$ and Kim SG: Effect of hyperoxia, hypercapnia, and hypoxia on cerebral interstitial oxygen tension and cerebral blood flow. Magn Reson Med 45: 61-70, 2001.

23. Rossi S, Stocchetti N, Longhi L, Balestreri M, Spagnoli D, Zanier ER and Bellinzona G: Brain oxygen tension, oxygen supply, and oxygen consumption during arterial hyperoxia in a model of progressive cerebral ischemia. J Neurotrauma 18: 163-174, 2001.

24. Ferrante F, Fusco E, Calabresi P and Cupini LM: Phytooestrogens in the prophylaxis of menstrual migraine. Clin Neuropharmacol 27: 137-140, 2004.
25. Claydon VE, Norcliffe LJ, Moore JP, Rivera M, Leon-Velarde F, Appenzeller $\mathrm{O}$ and Hainsworth R: Cardiovascular responses to orthostatic stress in healthy altitude dwellers, and altitude residents with chronic mountain sickness. Exp Physiol 90: 103-110, 2005.

26. Shmuel A, Yacoub E, Pfeuffer J, Van de Moortele PF, Adriany G, $\mathrm{Hu} \mathrm{X}$ and Ugurbil K: Sustained negative BOLD, blood flow and oxygen consumption response and its coupling to the positive response in the human brain. Neuron 36: 1195-1210, 2002.

27. Robertson CS, Gopinath SP, Valadka AB, Van M, Swank PR and Goodman JC: Variants of the endothelial nitric oxide gene and cerebral blood flow after severe traumatic brain injury. J Neurotrauma 28: 727-737, 2011

28. Ahnstedt H, Stenman E, Cao L, Henriksson M and Edvinsson L: Cytokines and growth factors modify the upregulation of contractile endothelin ET(A) and ET(B) receptors in rat cerebral arteries after organ culture. Acta Physiol (Oxf) 205: 266-278, 2012.

29. Xu X, Wang Z, Li Q, Xiao X, Lian Q, Xu W, Sun X, Tao H and Li $\mathrm{R}$ : Endothelial nitric oxide synthase expression is progressively increased in primary cerebral microvascular endothelial cells during hyperbaric oxygen exposure. Oxid Med Cell Longev 2: 7-13, 2009.

30. Simão F, Pagnussat AS, Seo JH, Navaratna D, Leung W, Lok J, Guo S, Waeber C, Salbego CG and Lo EH: Pro-angiogenic effects of resveratrol in brain endothelial cells: Nitric oxide-mediated regulation of vascular endothelial growth factor and metalloproteinases. J Cereb Blood Flow Metab 32: 884-895, 2012.

31. Willie CK, Macleod DB, Shaw AD, Smith KJ, Tzeng YC, Eves ND, Ikeda K, Graham J, Lewis NC, Day TA, et al: Regional brain blood flow in man during acute changes in arterial blood gases. J Physiol 590: 3261-3275, 2012. 